\title{
Registries and Cohorts to Accelerate Early Phase Alzheimer's Trials. A Report from the E.U./U.S. Clinical Trials in Alzheimer's Disease Task Force
}

\author{
P. Aisen', J. Touchon', S. Andrieu ${ }^{3,4,5,6,}$ M. Boada7, R. Doody', R.L. Nosheny ${ }^{9}$, J.B. Langbaum ${ }^{10}$, L. Schneider11, \\ S. Hendrix 12, G. Wilcock13, J.L. Molinuevo14, C. Ritchie15, P.-J. Ousset16, J. Cummings'17, R. Sperling18, \\ S.T. DeKosky19, S. Lovestone13, H. Hampel20,21, R. Petersen22, V. Legrand23, M. Egan24, C. Randolph25, \\ S. Salloway ${ }^{26}$, M. Weiner ${ }^{9}$, B. Vellas $3,4,5$ and Task Force Members*
}

*E.U./U.S. CTAD TASK FORCE: Wolfgang Barth (Lausanne), Russell Barton (Indianapolis), Christin Belexius (Basel), Gene Bowman (Lausanne), Sasha Bozeat (Utrecht), Robert Brashear (Fremont), Marc Cantillon (Livingston), Isabelle Clavier (Chilly Mazarin), Caroline Cohen (Chilly Mazarin), Preciosa Coloma (Basel), Johanna Carmel Egan (Indianapolis), Laura Eggermont (Utrecht), Howard Feldman (Vancouver), Phyllis Ferrell (Indianapolis), Laura Gault (North Chicago), Bjorn Gronning (Valby), Robert Hoerr (Karlsruhe), Lynne Hughes (Berks), Michael Irizarry (Indianapolis), Teresa Leon Colombo (Madrid), Johan Lindborg (Cambridge), Andrew Lockhart (Cambridge), Annette Merdes (Munich), Philip Nichols (Cambridge), Petr Novak (Bratislava), Matej Ondrus (Bratislava), Maria Pueyo (Suresnes), Rema Raman (San Diego), Michael Ropacki (Half Moon Bay), Rachel Schindler (New York), Peter Schueler (Langen), Märta Segerdahl Storck (Valby), Joao Siffert (Aliso Viejo), Jillian Smith (Welwyn Garden City), Andrew Stephens (Berlin), Joyce Suhy (Newark), George Vradenburg (Washington), Jens Wendland (Cambridge)

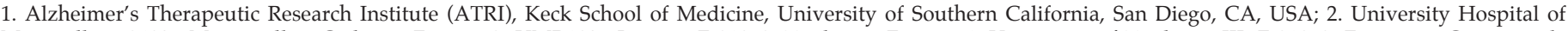

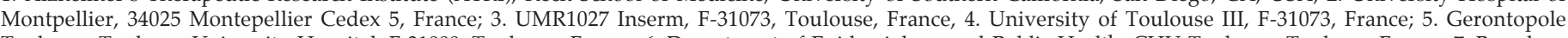

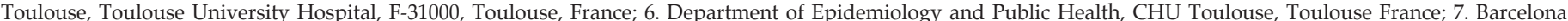

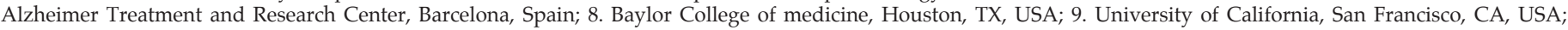

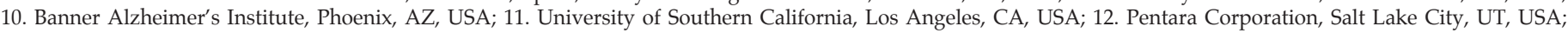

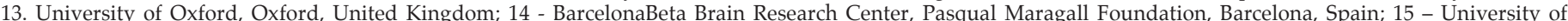

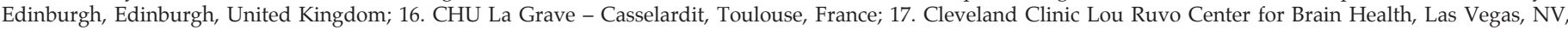

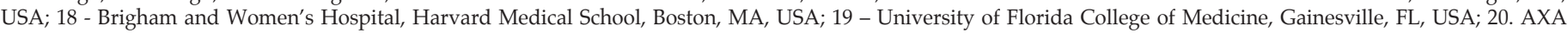

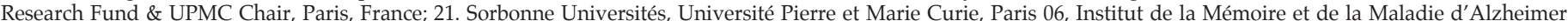

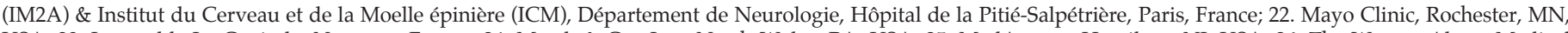

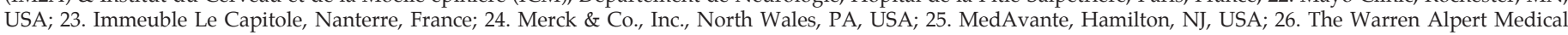
School of Brown University, Providence, RI, USA

Corresponding Author: Paul Aisen, Alzheimer's Therapeutic Research Institute (ATRI), Keck School of Medicine, University of Southern California, San Diego, CA, USA

\begin{abstract}
The EU/US/CTAD Task Force, an international collaboration of AD investigators from industry and academia, met in Barcelona, Spain, on November 4th, 2015, to explore existing and planned patient registries and other clinical trial infrastructure meant to expedite recruitment of large numbers of participants into clinical trials and improve their productivity. The Task Force identified a number of approaches currently being tested around the world, including the use of predictive algorithms to identify individuals likely to have prodromal or preclinical $\mathrm{AD}$, the establishment of clinical trial networks to streamline trials, and reforming the informed consent process to make it less burdensome to both investigators and trial participants. Multi-national systems such as the European Prevention of Alzheimer's Dementia (EPAD) and the Global Alzheimer's Platform (GAP) offer value for sponsors, trial sites, and patients by optimizing efforts to find effective disease-modifying and symptomatic treatments.
\end{abstract}

Key words: Alzheimer's disease prevention, patient registries, predictive algorithms, clinical trial networks, patient engagement.

\section{Introduction}

A $s$ the Alzheimer's disease (AD) field moves towards prevention (1), the challenge of bringing willing participants to research sites for early intervention trials has become a major bottleneck. Each new clinical trial, particularly those that target preclinical and prodromal populations, may take as long as three years to complete enrollment, with screen-fail rates as high as $90 \%$. Optimizing the design of networks and sites is also critical given the special expertise and infrastructure required to conduct biomarker and other assessments $(2,3)$. These challenges require not only novel approaches to trial design, but broader understanding and improved relationships among patients, caregivers, trial sites, and industry sponsors (2).

Barriers to increasing enrollment include low awareness on the part of patients and physicians of opportunities or suitability for such trials, uncertainty about participating in research, concerns about privacy, the time commitment of studies, and lack of access to the 
internet. Minority populations have been particularly difficult to enroll in clinical trials and may have concerns that differ from the majority population and require special attention especially regarding trust.

Over the past several years, and particularly since the G8 meeting in London in 2012, there has been increased interest in the idea of establishing registries of potential clinical trial candidates to provide the large number of participants that will be needed for upcoming pivotal trials. The G8 meeting also highlighted the necessity for a global approach. Thus, the EU/US/CTAD Task Force, an international collaboration of $\mathrm{AD}$ investigators from industry and academia, met in Barcelona on November 4th, 2015, to evaluate the current state of patient registries and other clinical trial infrastructure, identify gaps, and explore ideas for using and extending these resources to increase the productivity of clinical trials.

\section{Registries and Cohorts - existing and planned}

Traditionally, each clinical trial recruits participants separately, excluding those participants who do not meet the criteria for a particular study at a particular time. A registry, in contrast, provides a pool of individuals across a wide spectrum of disease risk, screened to varying degrees. Based on the needs of a particular trial, registrants may be selected for further screening or assessment and/or offered the opportunity to participate in the study; while those who don't meet those particular criteria remain in the registry for possible future studies. Meanwhile, the registry may continue to collect longitudinal health data and sometimes biospecimens from individuals. A cohort, by contrast, comprises a set of people with defined characteristics followed for a period of time to study longitudinal changes in their health or disease risk (4); this is the common methodology of large-scale epidemiological studies. The costs related to characterizing and following a cohort tend to be higher than the costs of constructing a basic registry. Thus, cohorts can be derived from registries, by selecting individuals who meet certain pre-determined criteria and enrolling them in an observational study.

Cohorts offer the potential for deep phenotyping and acquiring valuable longitudinal data, while registries are seen as an efficient way of identifying large numbers of potential trial subjects at a central location and collecting information that will allow them to be funneled to appropriate trials. Unlike registries in which the opportunity for participation in a clinical trial is a reason (and potential advantage) of enrolling, enrollees in epidemiological cohorts usually agree to be followed, observed, and perhaps biologically sampled but may be less eager to participate in clinical trials. Advantages of registries also include their accessibility to the public as well as health care professionals, their ability to draw participants from other databases (including existing cohorts), and their relatively low cost.

Registries and cohorts both have disadvantages as well. The large size and easy accessibility of registries results in the inclusion of many individuals not eligible for any anticipated studies, as well as the input of unedited and unchecked data, confidentiality concerns, and difficulty keeping data up to date. Registries also must balance their limited resources between recruitment, retention, and engagement. Cohorts are costly to establish and maintain, requiring substantial expertise for assessments and follow up. Incentives may be required to keep participants enrolled.

A number of AD-related registries have been established, each with its own set of goals (Table 1). These goals inform the approach each registry uses to reach out to potential registrants and the amount of information required to enroll. Some registries focus on getting the maximum number of people into the registry; others on getting as much information as possible.

For example, the Alzheimer's Prevention Registry (APR) (5), launched by the Banner Alzheimer's Institute in 2012 as a shared resource for the scientific community,

Table 1. Ongoing and Planned Alzheimer's Disease Registries

\begin{tabular}{|c|c|c|}
\hline Registry & Method of engagement & Population \\
\hline Alzheimer's Prevention Registry (APR) & $\begin{array}{l}\text { Internet-based recruitment through } \\
\text { information sharing }\end{array}$ & Normal age $18+$ \\
\hline Brain Health Registry & $\begin{array}{l}\text { Internet-based recruitment and } \\
\text { longitudinal monitoring }\end{array}$ & Normal; potentially MCI \\
\hline $\begin{array}{l}\text { Global Alzheimer's Platform (GAP) } \\
\text { Registry }\end{array}$ & $\begin{array}{l}\text { Recruitment from existing registries (e.g., } \\
\text { APR, BHR) }\end{array}$ & $\begin{array}{l}\text { Initially normal; may expand to other } \\
\text { populations }\end{array}$ \\
\hline $\begin{array}{l}\text { Cleveland Clinic Registry - } \\
\text { Healthybrains.org }\end{array}$ & Single center-based recruitment & Normal and $\mathrm{MCI} / \mathrm{AD}$ \\
\hline $\begin{array}{l}\text { Baylor College of Medicine Prevention } \\
\text { Registry }\end{array}$ & Single center registry & Normal age $60+$ \\
\hline
\end{tabular}


aims to accelerate enrollment into studies by providing sponsors and clinical investigators access to a pool of motivated, interested, and engaged participants. Modeled after other registries established to identify participants for studies of other conditions, such as the Army of Women for breast cancer (6) and the Fox Trial Finder for Parkinson disease (7), APR requires very little information at sign up - only a name, email address, zip/ postal code and year of birth - but encourages registrants to provide optional demographic information such as sex, race/ethnicity, and family history of AD. The registry then focuses on communication and engagement to educate registrants about research studies in general and study opportunities for which they might be candidates. APR currently has over 210,000 enrollees and continues to grow.

APR is currently helping to recruit subjects for 25 studies, with new studies being added every month. For example, after they sent an email to registrants about the Anti-Amyloid Treatment in Asymptomatic Alzheimer's (A4) study, there was an $89 \%$ spike in traffic to the website (8). They have also helped recruit for other registries such as the Brain Health Registry (BHR) (9), which reported that $7 \%$ of their membership came through APR.

The Brain Health Registry is a University of California, San Francisco-based registry that has the capacity to collect substantially more information at enrollment than APR, as its goal is to provide screening and longitudinal assessment of individuals for potential participation in clinical trials of $\mathrm{AD}$ and other neurological diseases. Participants must sign an online consent to participate; they then have the option of completing a number of self-report questionnaires, based on well-validated instruments (10-16) that assess cognitive function and include common inclusion and exclusion criteria for AD trials. BHR participants can also take online neuropsychological tests that measure cognitive function across multiple domains, including memory, learning, attention, and processing speed (17-23), and are asked to return to the website every six months for followup online assessments, generating a large database of longitudinal data that can be used to identify those undergoing cognitive decline. At the time of the Task Force meeting, over 31,000 participants had enrolled, about half with memory complaints and about onequarter with a family history of $\mathrm{AD}$. The retention rate for one-year follow-up was $42 \%$, demonstrating the feasibility of collecting longitudinal data from a large cohort online. The BHR is actively referring participants into clinical studies. A the time of the Task Force meeting, over 1600 BHR participants had been referred to specific studies, and over 150 had been seen in clinics.

The European Prevention of Alzheimer's Dementia (EPAD) project seeks to build a platform across Europe for more effective delivery of Phase 2 Proof of Concept studies. There are 3 key elements; the EPAD Registry, the
EPAD Longitudinal Cohort Study and the EPAD Proof of Concept Trial. All 3 elements are being effectively managed within the same program (24). The EPAD registry uses a different approach to acquire participants, selecting existing parent cohorts from across Europe for inclusion. Then, they use a data discovery/sharing tool, which enables data to be queried by EPAD investigators while it remains within the parent cohort. This model allows each cohort to maintain its own "rules of engagement" while still participating in this panEuropean initiative. For example, the UK Biobank does not allow disclosure of genotype, so EPAD investigators would be allowed to contact participants only if they have a protocol that ensures no inadvertent disclosure.

The French EPAD Registry uses yet another approach to enroll participants and gather data. Their Memory Clinic-Based Registry, based on the principle that bridging the gap between research and clinical care is necessary to promote participation in clinical trials (25), aims to establish a registry when consultations and evaluations are carried out in clinical practice. At each of 14 centers of excellence across France, screening of clinic patients is based on three simple requirements: 1) availability of a caregiver or informant, 2) sufficient participant autonomy to attend trial visits, and 3) no major contraindication or exclusionary factors. If all three of these requirements are met, the participant and caregiver are asked if they agree to participate in a clinical trial. If they respond affirmatively, clinic staff check for available trials, verify inclusion and exclusion criteria and refer to appropriate trials that match the participant's characteristics. If no appropriate trials are available, the participant enters a follow-up cohort with reevaluations every six months.

For EPAD, the target population is non-demented participants 50 years of age or older who are deemed to be at risk for $\mathrm{AD}$ based on memory complaint and the presence of $\mathrm{MCI}$, prodromal $\mathrm{AD}$, or positive family history. Between March and October 2015, the 14 French EPAD sites recruited 2,952 subjects and began ongoing data collection, indicating that memory clinic-based registries represent an easy-to-implement and flexible recruitment source for clinical trials.

The Global Alzheimer's Platform (GAP) Registry is one element of an overall initiative (GAP) established to achieve in North America what EPAD aims to achieve in Europe. The GAP registry, still in development, will tap existing registries including BHR, APR, and others to enroll non-demented participants over age 50 as well as study partners. They will obtain electronic consent that indicates their interest in future trials and gives permission for their information to be used to determine eligibility for such trials. The registry will collect demographic data as well as data from subjective and objective cognitive assessments and DNA. A subset of registrants will be selected for in-clinic testing. From this registry, a trial-ready cohort will be established as 
described below.

The Cleveland Clinic has offered a very different type of registry, a web-based portal called Healthybrains. org, which aims to reduce the risk of late-life cognitive decline through education about a brain-healthy lifestyle. Participants provide data on a number of lifestyle factors that influence risk and that are under their personal control, from which is calculated a Brain Health Index. The site also collects other data, including demographics, cognitive function, a memory self-rating, family history, medical diagnoses (if any), and the presence of other risk factors such as cardiovascular disease. A questionnaire is used to gauge interest in clinical trials and offers the option of signing up for clinical trials. Those who agree to be contacted about trials are connected to either the Cleveland Clinic Lou Ruvo Center for Brain Health if they are Las Vegas residents; all others are referred to the Alzheimer's Association's TrialMatch (26). Preliminary results indicate that of 3,103 active registrants, more than half expressed interest in clinical trials, nine have been screened, one randomized, and 150 are pending screening. Ultimately, they intend to feed participants into the GAP registry. However, one concern raised by Task Force members was that by suggesting that a brain-healthy lifestyle can prevent $\mathrm{AD}$, individuals may not see the value of participating in clinical trials. To avoid this, participants will need to be educated about the limitations of the healthy lifestyle approach and the observation that those who participate in clinical trials decline less over time than those not in trials.

The Baylor College of Medicine also recently established a prevention registry, targeting individuals 60 years and older through postcards sent to strategic zip codes, as well as patients at the Baylor clinic. Of the first 85,000 cards sent, about $1.5 \%$ responded and subsequently received a phone call and questionnaire. In a short time, over 800 participants were identified as ready to enter prevention trials. At the time of the Task Force meeting, over 100 had been enrolled in the TOMMORROW trial and about 20 had been screened for A4. Only an estimated $30 \%$ of all potentially eligible subjects have been contacted with the first mailing in the Greater Houston area.

\section{Moving from registries to cohorts to drug trials}

Registries are just one part of an overall strategy to increase recruitment in clinical trials. While registries offer the potential to speed trial subject identification, initiating the study requires additional steps including screening, obtaining consent for trial participation, and setting up a trial site capable of providing all of the resources and tools required by the trial. GAP and EPAD both include multiple workstreams to promote clinical trial efficiency from the time of enrollment in a registry through the completion of the trial. One GAP initiative will enable the development of Trial Ready Cohorts for the Prevention of Alzheimer's Dementia (GAPTRC PAD), which initially will include 1,000 preclinical and 1,000 prodromal subjects that have been pre-screened and are ready to enroll in trials. Participants for the cohort will be selected using an adaptive algorithm to predict amyloid status, which is currently being developed and validated (ref Sperling paper in this issue).

BHR also has applied a hierarchical approach to criteria to identify subjects for preclinical and prodromal studies. As illustrated in Figure 1, from the original group of 31,428 participants, $1,810(5.8 \%)$ are considered likely candidates for prodromal studies based on their cognitive test scores, age, and self-reported memory concerns; as well as their lack of exclusionary criteria and their agreement to be contacted for a study. Using more stringent criteria, such as family history of $\mathrm{AD}$ and selfreported mild cognitive impairment (MCI), the cohort can be further reduced to 144 participants. Using less strict criteria would likely result in a higher number of screen failures, while the more stringent criteria might exclude eligible participants and decrease the needed number of cases. Geography must also be factored in if the trial is limited to those in a circumscribed area.

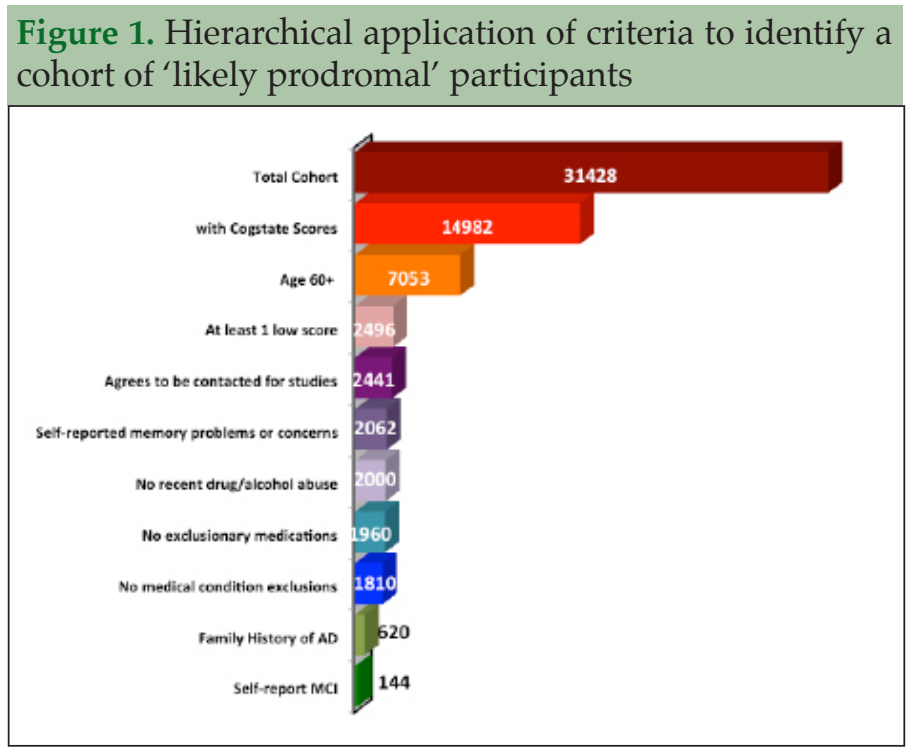

A similar hierarchical approach was used to identify $5,098(16.2 \%)$ of the 31,428 participants in the BHR registry as "likely preclinical AD." BHR then developed an enrichment algorithm, based on data from the Alzheimer's Disease Neuroimaging Initiative (ADNI), to enrich this group for amyloid positivity. For this algorithm, amyloid positivity was predicted by age, scores on auditory-verbal learning test (AVLT) and family history of $\mathrm{AD}$, and resulted in the identification of $14.6 \%$ of the 'likely preclinical' cohort as likely to be amyloid positive (Nosheny 2015 paper in this issue of JPAD). This analysis demonstrates the potential to use non-invasive means to select likely amyloid positive 
participants who could be screened subsequently with amyloid PET imaging studies for trials requiring amyloid positivity, thus reducing the number of screen failures as well as study costs. However, it also demonstrates that a very large number of candidates will be needed to identify those eligible for such a study. Concerns were also raised about the specificity of the algorithm derived from ADNI data, and its potential to exclude subjects unnecessarily. Data from other cohorts, such as the Australian Imaging, Biomarkers, and Lifestyle Study (AIBL), may help to further refine the algorithm.

\section{Increasing recruitment in $\mathrm{AD}$ trials}

Barriers to clinical trial recruitment vary across the spectrum of disease severity and as a result of idiosyncratic issues that characterize each trial. For example, one of the trials conducted by the Alzheimer's Prevention Initiative (API) and another by the Dominantly Inherited Alzheimer's Network Trials Unit (DIAN-TU) target individuals with autosomal dominant mutations that cause AD in $100 \%$ of carriers; these individuals represent only about $1 \%$ of all AD cases. A second API study targets those who are homozygous for APOE 4 , which markedly increases their risk of developing AD (27); only $2 \%$ of the population falls into this category. A4 targets asymptomatic individuals who are amyloid positive, which represents about $30 \%$ of those aged 65 or older, and the percentage who are positive increase with age, approaching $50 \%$ in the mid80 s.

The algorithm and hierarchical approach mentioned earlier should help investigators identify amyloid positive individuals for the A4 trial as well as other trials of amyloid-targeting agents (28). Meanwhile, APR is about to spin off a separate IRB-approved program called GeneMatch (29), the goal of which is to match individuals to AD studies based in part on their APOE genotype. For this program, interested individuals are shown a short, interactive educational video that provides information about Alzheimer's, APOE, and the GeneMatch program. After the video, participants are directed to the informed consent, which is signed electronically. They are then provided with a swab kit for them to submit DNA. Participants are not told their APOE genotype and invitations to participate in a study must not inadvertently disclose genotype.

Gaining the enthusiastic participation of general practitioners is also essential to promote trial enrollment, particularly since by the time a patient sees a specialist, the disease may be so advanced that treatments will have little impact. In the United States, there is a move towards using metrics to tie reimbursement rates to an assessment of physicians' practice. Similar to good clinical practice in oncology, we suggest that physician encouragement to participate in clinical trials should be viewed as a part of good clinical care and used as one of these metrics.
In Toulouse, France, the limited participation of general practitioners has been addressed by sending nurses from Gerontopole into family practice offices to do assessments. Not only does this relieve the burden on the family practitioners, but it also trains them in how to assess patients for cognitive impairment. Following the assessment, the nurses meet with the physicians to describe existing trials for which their patients may be eligible. Programs such as this are probably best suited for areas that have strong links between hospitals and community-based medicine, and are close enough to trial sites to make participation feasible for volunteers. While some Task Force members thought that such programs might meet with much greater resistance in the United States, not all U.S. members agreed; however, all agreed that it would be worth exploring. In Wisconsin, a network of primary care physicians regularly refer patients to the University of Wisconsin, Madison for clinical research studies, but whether that has improved recruitment into clinical trials was not known.

Part of the reason for lack of patients enrolled in trials is the lack of enthusiastic participation of primary care physicians - general practitioners, family practitioners, and geriatricians. Indeed, this reluctance to pursue clinical trials extends even into registry recruitment. Attempts by APR to send direct messages to physicians encouraging their patients to enroll resulted in very few sign ups. However, primary care physicians also may need to be assured that enrollment in a clinical trial will not lead to the patient leaving that physician's s practice.

An important issue for many patients with regard to participation in clinical trials is the concern that they will receive placebo rather than active drug. Unbalanced randomization may help alleviate this problem; the difference between a $50 \%$ and a $66 \%$ chance of getting a drug can make a big difference in how patients and families view the trial. Open label extensions where the drug is offered to $100 \%$ of trial participants may also help, although with very long trial, even this may be insufficient since patients receiving placebo would have to wait a considerable length of time before receiving the drug. Here the emphasis must be that the study medication cannot be obtained any other way, and the increased medical care and observation provided by trial participation, which is free to the family, is a significant benefit.

\section{Improving the productivity of clinical trial centers}

Merely accelerating recruitment for clinical trials will not address all the challenges that have led to low productivity in AD trials (30). Thus, EPAD, GAP, and other initiatives encompass multiple broad efforts. For example, GAP-NET, one of GAP's four workstreams, comprises a standing network of clinical trial sites with 
continuous budgeting and facilitated administrative processes to reduce the sites' start-up time, a process that can delay substantially the initiation of trials. GAP NET plans to conduct trials for symptomatic and diseasemodifying compounds across the continuum of disease severity. Eleven pilot sites have been selected, with 100 sites planned.

In the United States, another network has been established by the Patient-Centered Outcomes Research Institute (PCORI), an independent nonprofit agency authorized by the Patient Protection and Affordable Care Act in 2010. This network, called the National Alzheimer's and Dementia Patient and Caregiver-Powered Research Network (AD PCPRN), is a collaboration among the Mayo Clinic, US Against Alzheimer's, the University of Florida, and the BHR. By bringing together the existing patient-centered, web-based registries of these partners using a common data set, the PCPRN aims to amplify the patient and caregiver voice in efforts to accelerate the development of effective treatments for $\mathrm{AD}$ and other dementias. AD PCPRN has a particular focus on minority recruitment. With a three-year, \$1.56 million contract from PCORI, the network will fund a variety of research projects, including a project aiming to enhance enrollment in clinical trials and another that will assess biomarker findings in community-based, as opposed to research center populations.

Streamlining the informed consent process to make it less burdensome to both investigators and trial participants is also viewed as essential to improving the productivity of clinical trials (31). Reforming the process through the use of electronic informed consent (eIC), using multiple forms of electronic media, has been supported by regulatory agencies, including the FDA (32). Not only does eIC potentially reduce burden, but it also can be tailored to ensure that participants fully understand the information presented and that the consent form is completed accurately. Improving site-tosite and inter-rater consistency through the use of audio recording and central review of outcome assessments was also discussed by the Task Force as a step towards improving data validity and reliability, as well as the power (and thus the cost) of clinical trials (33).

\section{Moving forward - improving accuracy, compliance, retention}

Task Force participants reporting on these registries outlined a number of key issues important to consider when planning future registry efforts:

- Engagement of patients and caregivers is crucial and requires multiple touchpoints and personalization to make participants feel more connected.

- Online targeted advertising is likely to generate more signups than giving talks at community events.

- General practitioners need to be engaged as partners, with approaches that assure no loss of their patients and seeing trials as a benefit to their patients.

- Registries that monitor decline in performance need to identify best practices that encourage people to do repeated online assessments, such as rewards or providing feedback and a sense of progress.

- Prescreening methodology should aim to identify people who want to be in clinical trials and will stay with a trial through completion.

Integrated systems such as GAP and EPAD offer value across all stakeholder groups. For patients, therapies are likely to reach the market more quickly. For trial sites, stable funding and eliminating the need for repetitive training on the same tools will increase efficiency. For sponsors, the time savings translate into reduced costs and the ability to test more compounds. Task Force members predicted that in the near future it will become commonplace for people to consider participating in a registry, cohort, or trial, but warned that any large-scale data breach would set back the effort enormously.

For these systems to achieve their full potential in advancing drugs to patients, they must be combined with enhanced discovery. Task Force members agreed that the field should try to emulate what hs been done in cancer, where clinical research is more integrated into clinical practice. Moreover, the field needs to prepare for the emergence of precision medicine approaches and an increased focus on patient-centered care. As these efforts gain momentum, a major concern will be how to share data with people based on their level of interest, and how much information needs to be provided about the meaning of those data for individuals with their own unique genetics, environment, and expectations.

Acknowledgements: The authors thank Lisa J. Bain for assistance in the preparation of this manuscript.

Conflict of interest: Authors have no conflicts of interest with this paper. The Task Force was partially funded by registration fees from industrial participants. These corporations placed no restrictions on this work.

\section{References}

1. Vellas B, Bateman R, Blennow K, Frisoni G, Johnson K, Katz R, et al. Endpoints for Pre-Dementia AD Trials: A Report from the EU/US/CTAD Task Force. J Prev Alzheimers Dis. 2015;2(2):128-35.

2. Hampel H, Frank R, Broich K, Teipel SJ, Katz RG, Hardy J, et al. Biomarkers for Alzheimer's disease: academic, industry and regulatory perspectives. Nat Rev Drug Discov. 2010;9(7):560-74.

3. Cavedo E, Lista S, Khachaturian ZS, Aisen PS, Amouyel P, Herholz K, et al. The road ahead to cure Alzheimer's Disease: Development of biological markers and neuroimaging methods for prevention trials across all stages and target populations. J Prev Alz Dis. 2015.

4. Morabia A. A History of Epidemiologic Methods and Concepts. Basel: Birkhaeuser Verlag; 2004.

5. ClinicalTrials.gov. Identifier: NCT02022943. Available from: clinicaltrials.gov.

6. Susan Love Research Foundation. Army of Women. Available from: https:// www.armyofwomen.org/.

7. Michael J. Fox Foundation. FoxTrialFinder. Available from: https:// foxtrialfinder.michaeljfox.org/.

8. Alzheimer's Therapeutic Research Institute. The A4 Study. Available from: http: / / a4study.org/.

9. The Regents of the University of California. Brain Health Registry. 2012-2015.

10. Buysse DJ, Reynolds CF, 3rd, Monk TH, Berman SR, Kupfer DJ. The Pittsburgh Sleep Quality Index: a new instrument for psychiatric practice and 
research. Psychiatry Res. 1989;28(2):193-213

11. Corrigan JD, Bogner J. Initial reliability and validity of the Ohio State University TBI Identification Method. J Head Trauma Rehabil. 2007;22(6):31829.

12. Farias ST, Mungas D, Reed BR, Cahn-Weiner D, Jagust W, Baynes K, et al. The measurement of everyday cognition (ECog): scale development and psychometric properties. Neuropsychology. 2008;22(4):531-44.

13. King NS, Crawford S, Wenden FJ, Moss NE, Wade DT. The Rivermead Post Concussion Symptoms Questionnaire: a measure of symptoms commonly experienced after head injury and its reliability. J Neurol. 1995;242(9):587-92.

14. von Steinbuechel N, Wilson L, Gibbons H, Muehlan H, Schmidt H, Schmidt S, et al. QOLIBRI overall scale: a brief index of health-related quality of life after traumatic brain injury. J Neurol Neurosurg Psychiatry. 2012;83(11):10417.

15. Ware JE, Jr., Sherbourne CD. The MOS 36-item short-form health survey (SF36). I. Conceptual framework and item selection. Medical care. 1992;30(6):47383.

16. Yesavage JA, Brink TL, Rose TL, Lum O, Huang V, Adey M, et al. Development and validation of a geriatric depression screening scale: a preliminary report. J Psychiatr Res. 1982;17(1):37-49.

17. Chen KH, Chuah LY, Sim SK, Chee MW. Hippocampal region-specific contributions to memory performance in normal elderly. Brain and cognition. 2010;72(3):400-7.

18. Fredrickson J, Maruff P, Woodward M, Moore L, Fredrickson A, Sach J, et al. Evaluation of the usability of a brief computerized cognitive screening test in older people for epidemiological studies. Neuroepidemiology. 2010;34(2):6575.

19. Lim YY, Ellis KA, Harrington K, Ames D, Martins RN, Masters CL, et al. Use of the CogState Brief Battery in the assessment of Alzheimer's disease related cognitive impairment in the Australian Imaging, Biomarkers and Lifestyle (AIBL) study. J Clin Exp Neuropsychol. 2012;34(4):345-58.

20. Lim YY, Jaeger J, Harrington K, Ashwood T, Ellis KA, Stoffler A, et al. Threemonth stability of the CogState brief battery in healthy older adults, mild cognitive impairment, and Alzheimer's disease: results from the Australian Imaging, Biomarkers, and Lifestyle-rate of change substudy (AIBL-ROCS). Archives of clinical neuropsychology : the official journal of the National Academy of Neuropsychologists. 2013;28(4):320-30.

21. Mathewson KJ, Dywan J, Snyder PJ, Tays WJ, Segalowitz SJ. Aging and electrocortical response to error feedback during a spatial learning task. Psychophysiology. 2008;45(6):936-48.
22. Pietrzak RH, Maruff P, Snyder PJ. Convergent validity and effect of instruction modification on the groton maze learning test: a new measure of spatial working memory and error monitoring. Int J Neurosci. 2009;119(8):1137-49.

23. Sternberg DAH, Joseph L; Katz, Ben; Ballard, Kacey; Scanlon, Michael, editor The Brain Performance Test: Preliminary Findings of transfer from cognitive training to a repeatable, dynamically generated assessment. Society for Neuroscience; 2012; New Orleans, LA.

24. Ritchie CW, Molinuevo JL, Truyen L, Satlin A, Van der Geyten S, Lovestone $S$, et al. Development of interventions for the secondary prevention of Alzheimer's dementia: the European Prevention of Alzheimer's Dementia (EPAD) project. Lancet Psychiatry. 2015.

25. Watson JL, Ryan L, Silverberg N, Cahan V, Bernard MA. Obstacles and opportunities in Alzheimer's clinical trial recruitment. Health Aff (Millwood). 2014;33(4):574-9.

26. Alzheimer's Association. TrialMatch. Available from: http://www.alz.org/ research/clinical_trials/find_clinical_trials_trialmatch.asp.

27. Corder EH, Saunders AM, Strittmatter WJ, Schmechel DE, Gaskell PC, Small GW, et al. Gene dose of apolipoprotein E type 4 allele and the risk of Alzheimer's disease in late onset families. Science. 1993;261(5123):921-3.

28. Aisen PS, Vellas B, Hampel H. Moving towards early clinical trials for amyloid-targeted therapy in Alzheimer's disease. Nat Rev Drug Discov. 2013;12(4):324.

29. ClinicalTrials.gov. Identifier: NCT02564692.

30. Paul SM, Mytelka DS, Dunwiddie CT, Persinger CC, Munos BH, Lindborg SR, et al. How to improve R\&D productivity: the pharmaceutical industry's grand challenge. Nat Rev Drug Discov. 2010;9(3):203-14

31. Nishimura A, Carey J, Erwin PJ, Tilburt JC, Murad MH, McCormick JB Improving understanding in the research informed consent process: a systematic review of 54 interventions tested in randomized control trials. BMC Med Ethics. 2013;14:28.

32. Food and Drug Administration. Use of Electronic Informed Consent in Clinical Investigations: Questions and Answers. Guidance for Industry. Draft Guidance. In: CDER O, CBER, CDRH, editor. 2015.

33. Muller MJ, Szegedi A. Effects of interrater reliability of psychopathologic assessment on power and sample size calculations in clinical trials. J Clin Psychopharmacol. 2002;22(3):318-25. 